\title{
TRABAJANDO DE FORMA COOPERATIVA EN LA ESCUELA: ABRIENDO LA PUERTA A LAS FAMILIAS
}

\author{
WORKING IN A COOPERATIVE WAY AT SCHOOL: OPENING THE DOOR TO \\ FAMILIES
}

Marina García Carmona (marinagc@ugr.es). Becaria FPU. Programa de Doctorado Currículum, Profesorado e Instituciones Educativas. Universidad de Granada

\author{
Fecha de recepción: 28 de mayo de 2012 \\ Fecha de aceptación: 11 de junio de 2012 \\ Localizador: http:www.ugr.es/local/miguelgr/ReiDoCrea-Vol.1-Art.8-Garcia.pdf
}

\section{Resumen}

Este estudio pretende mostrar la importancia del trabajo cooperativo en la escuela, en especial del relacionado con la participación de los padres y la familia de los alumnos con el profesorado y el centro educativo. Por otro lado, se propondrán variadas acciones y actividades educativas prácticas que se pueden llevar a cabo en el mismo para conseguir la relación cooperativa familia-escuela y alcanzar así el clima óptimo para el desarrollo integral del alumno y una educación de calidad que serán los principales objetivos perseguidos. Todo ello se hará en el marco del respeto y la integración de todos los sujetos implicados.

Palabras clave: Relación padres-escuela, familia, participación de los padres, enseñanza en equipo, cooperación educacional.

\begin{abstract}
This paper seeks to show the importance of cooperative work at school, especially in relation to parent and family participation with teachers and schools. We will also propose a variety of actions and practical educational activities that can be used at school in order to achieve a cooperative family-school relationship and to create an optimum environment for the development of the student and a quality education that will be the main goals pursued. The study will be carried out in a respectful and integrative manner for all subjects involved.
\end{abstract}

Keywords: Parent-school relationship, family, parent participation, team teaching, educational cooperation. 


\section{INTRODUCCIÓN}

En la sociedad cambiante en la que vivimos, se hace imprescindible la innovación y actualización de las instituciones educativas. El trabajo en equipo, que lucha por metas y finalidades comunes, es cada vez más frecuente en nuestras vidas y los alumnos deben aprender a cómo trabajar con sus compañeros para conseguir objetivos afines en el centro escolar y en su vida diaria.

La cooperación es un elemento básico para los centros educativos y, en este sentido, lo que en ellos solemos encontrar son prácticas e iniciativas en las que alumnado y profesorado cooperan: los primeros para aprender y los segundos para enseñar; al mismo tiempo, ambos colectivos, alumnos y profesores, cooperan unos con otros, y no sólo en el aula, sino también en otras instancias complementarias con el objetivo de ofrecer una enseñanza de calidad para todos (Durán y Miquel, 2003).

En todas las acciones educativas llevadas a cabo en la escuela se debe poner de manifiesto cómo es posible aprender a cooperar en el aula y cómo una organización cooperativa de la clase recupera en la cotidianidad de la vida diaria valores de convivencia democrática, tan en desuso en la actualidad. Proponer la cooperación como práctica educativa, en una situación de predominante individualismo como es el momento actual, es necesario para recuperar el valor formativo que la sociedad y la escuela en algunos momentos pasan desapercibido.

Todas estas palabras se llenan de sentido al hablar de las relaciones del colegio con los padres o familias de los alumnos. La coherencia de la educación llevada a cabo en las distintas aulas con la practicada en cada uno de los hogares de los alumnos, se consigue mediante una comunicación cooperativa entre ellos. Veremos, a continuación la importancia de dicha cooperación y las ventajas más destacadas encontradas en su práctica.

\section{APRENDIZAJE COOPERATIVO EN EQUIPO PARA LA MEJORA DE LA CALIDAD EDUCATIVA}

Se realiza un trabajo cooperativo cuando los objetivos de los participantes se hallan vinculados de tal modo que uno sólo puede de tal modo que uno sólo puede alcanzar sus objetivos si y sólo si los demás consiguen los suyos (Rué, 1989). Unos años después, el mismo autor en trabajos posteriores matiza lo dicho sobre esta temática: El término aprendizaje cooperativo es un término genérico usado para referirse a un grupo de procedimientos de enseñanza que parten de la organización de la clase en pequeños grupos mixtos y heterogéneos donde los alumnos trabajan conjuntamente de forma cooperativa para resolver tareas académicas (Rué, 1998:20).

Como podemos ver, el trabajo cooperativo debe ser llevado a cabo en el aula para que ésta sirva como vehículo transmisor de los valores que tiene como inherentes. La ayuda al compañero, la lucha por fines comunes, la empatía, la confianza y el compromiso por compartir, hacen que sirvan de ejemplo al alumno a la hora de enfrentarse a metas complejas.

También, fuera del ámbito educativo, la sociedad se manifiesta generalmente a favor de esta actividad que viene avalada por la idea de que la unión hace la fuerza mientras que el aislamiento provoca debilidad y sitúa al docente en condiciones de desventaja para enfrentarse a los retos que plantea la enseñanza (López Hernández, 2007:49).

El conjunto del alumnado, una vez haya pasado por la etapa escolar y durante la misma, deberá enfrentarse a la sociedad. Para ello necesitará diferentes herramientas 
sociales o de relación que irán encaminadas, en numerosos casos y para conseguir mayores metas, al trabajo en equipo.

Por otro lado, una educación cooperativa debe fijar reglas, organizar espacios e instrumentos, marcar objetivos, evaluar resultados, reflejarse en las estrategias, comparar las elecciones, y todo de manera colectiva (Campiglio y Rizzi, 1997). Para conseguirlo hace falta una adaptación progresiva de cada uno por separado a la acción común. Por consiguiente, es deber de la escuela llevar a cabo con sabiduría y profesionalidad la transformación de las múltiples experiencias en elaboraciones culturales formalizadas y concretamente en la capacidad de vivir al lado de personas diferentes.

Castelló (1998:59) nos habla de las situaciones cooperativas como aquellas en las que los recursos individuales se aúnan para conseguir objetivos comunes, a la vez que las presiones del grupo sobre sus componentes se orientan hacia la mejora de su rendimiento en beneficio del propio grupo, en contraposición con las situaciones competitivas en las que las presiones del grupo o las rivalidades entre subgrupos actúan como elemento motivador.

Este mismo autor, siguiendo a Gage y Berliner (1988), nos habla de algunas características que se suelen producir en las situaciones cooperativas (Castelló. 1998:59-60):

- Puesto que el resultado del proceso no se atribuye a individuos concretos, sino al grupo, el éxito o el fracaso también se reparte entre los componentes, aumentando las oportunidades de éxito y dando opciones para corregir las respuestas menos acertadas de los sujetos.

- Al aprovechar los recursos individuales, las aportaciones deben estar coordinadas, lo cual representa una dificultad adicional, pero cuando se afrontan tareas complejas, el trabajo en equipo aporta facilidades que son muy difíciles de encontrar de manera individual.

- Por otra parte, puesto que dentro de un grupo cooperativo se distribuyen las tareas a los alumnos, todos pueden aportar al grupo aquello que mejor hacen.

Como podemos observar, todas estas premisas son perfectamente extrapolables al aula. Los alumnos deberán trabajar por conseguir las mismas metas y este camino estará condicionado por las relaciones que en el grupo se establezcan. Vemos cómo la capacidad de ponerse en el lugar del otro resulta imprescindible.

Finalmente, añadir que el último fin de la cooperación educativa será en todo momento la formación global de una personalidad gracias a la cual todo individuo cooperante pueda desarrollar, mediante una productiva pertenencia al grupo, la capacidad de moverse y actuar dentro y fuera del mismo, con plena autonomía.

\section{LA IMPORTANCIA DEL TRABAJO COOPERATIVO ENTRE FAMILIAS $Y$ PROFESORADO}

Cada día más se habla del binomio familia-escuela como entidad formadora de los futuros ciudadanos y es por la mayoría sabido que, sin una buena relación que llene de cohesión las acciones educativas, no es posible el desarrollo integral de la persona (García Carmona y Sola Martínez, 2010:1).

En esta relación, basada primordialmente en la cooperación de las dos partes que forman el mencionado binomio, el profesorado juega un papel importantísimo ya que 
debe fomentar e inculcar esta cultura participativa a los padres y familiares de sus alumnos.

Por otro lado, debemos tener en cuenta que cuando existen finalidades comunes se crea un proceso de reciprocidad positiva que constituye otro requisito para la colaboración y que este tipo de relaciones se producen cuando los diferentes proyectos de los miembros del equipo se favorecen mutuamente (López Hernández, 2007:23).

Este tipo de finalidades se hacen palpables cuando a la educación de los alumnos o hijos se refieren. Tanto el profesorado como los padres y familias de los alumnos juegan un papel importantísimo en su educación. El entramado de relaciones deberá consistir en una reciprocidad positiva en la que se favorezcan entre sí y en la que velen por el desarrollo íntegro como persona del estudiante.

En este mismo sentido, la colaboración entre familia y profesorado, especialmente dentro del centro escolar, es una necesidad cada vez más patente y así lo entienden todos los sectores implicados en la enseñanza: los padres, los equipos directivos, el profesorado en general $\mathrm{y}$, de forma especial, la administración educativa (López Hernández, 2007:49).

En esta colaboración, la capacidad de empatía cobra vital relevancia a la hora de relacionarse. Un clima de confianza y respeto es fundamental para que la comunicación transcurra con éxito y se consigan las metas propuestas en aras a una educación de calidad y coherente.

Además, debemos destacar el rol que juega la educación inclusiva en estas relaciones. La creación del sentido de comunidad es un elemento esencial de la enseñanza inclusiva, y ese sentido se crea y se mantiene haciendo de la cooperación una suerte de actitud y de práctica "transversal" que se haga extensible también a las relaciones cruzadas entre profesores y alumnos o entre familias y profesores (Durán y Miquel, 2003). La educación inclusiva lucha por la integración de todas las personas independientemente de sus características personales o culturales. Debemos fomentar este espíritu y que, al igual que la inclusión se transforma en un elemento multidisciplinar, conseguir que la cooperación así lo haga también.

Resulta interesante resaltar en este momento el trabajo de Comellas (2009:54) donde la autora aporta una serie de temas de comunicación muy interesantes entre familia y escuela que pueden ayudar a guiar la relación entre ambos. Estos aspectos pueden ser tratados en reuniones en las que ambas partes estén representadas para llegar a acuerdos relacionados con las vidas de los alumnos en el centro escolar. Éstos pueden ser, entre otros:

- Ideario educativo de la escuela.

- Criterios educativos de la escuela y su puesta en práctica.

- Aspectos relacionados con la maduración de los niños.

- Aspectos relacionados con la cultura y la sociedad en la que nos encontramos.

- La educación en general. 
Veamos, a continuación algunos ejemplos de participación cooperativa de los padres y familias en la escuela.

\section{ACTIVIDADES QUE FOMENTAN LA COOPERACIÓN EN EL CENTRO ESCOLAR Y EN LAS AULAS}

Una vez que hemos visto algunas de las numerosas ventajas y finalidades de este trabajo cooperativo entre el profesorado y las familias para la mejora de la calidad educativa de los alumnos, presentaremos algunas acciones y actividades que facilitan dicha práctica y finalmente presentaremos una experiencia llevada en un centro escolar de la ciudad de Granada en la etapa de Educación Infantil. Éstas, entre otras, pueden ser:

a) Aprender sobre las familias de diferentes culturas mediante grupos de trabajo: podemos elaborar proyectos conjuntos en las clases en los que colaboren los padres y los alumnos (con la supervisión del profesor en todas las etapas) y que cada grupo se encargue de presentar una cultura diferente al resto de compañeros.

b) Uso de la agenda escolar: los padres y profesores pueden trabajar de forma conjunta manteniéndose comunicados, bien diariamente o bien semanalmente, mediante esta herramienta donde podrán ir comentándose las dificultades, logros o simplemente desarrollo del alumno.

c) Promover un sentido de comunidad entre las familias: a la vez que se mantiene relación con las diferentes familias de los alumnos, el profesorado debe fomentar también que se establezcan unas relaciones positivas entre los padres para que todos colaboren en la educación del alumnado y persigan las mismas metas.

d) Ayudar a que las familias con menores ingresos económicos se sientan miembros importantes de la comunidad educativa y ayuda a las mismas por parte de los padres y maestros, en la medida de las posibilidades de cada uno. Se deberá promover entre todas un clima de ayuda e integración de aquellos que más lo necesitan.

e) Proyecto de publicación de historias de los niños: los padres elaborarán un libro con las historias o redacciones que escriben los alumnos de la clase durante el período lectivo con la ayuda del profesor. Entre todos (padres, alumnos y profesores) se publicará un libro al final del curso escolar realizado por todos los padres y niños de la clase.

Para finalizar este apartado, presentaremos una actividad llevada a cabo en un colegio de Granada en el segundo ciclo de Educación Infantil. La experiencia es la titulada "el periódico-mural en la escuela infantil". En esta actividad los padres de los alumnos contaron y representaron diversos cuentos y después los estudiantes de los tres cursos del segundo ciclo de Educación Infantil dibujaron los personajes más significativos y elaboraron conjuntamente su periódico-mural. A través de las siguientes fotografías se puede observar cómo los niños han pegado en el mural los dibujos realizados por ellos mismos y en grupo: 


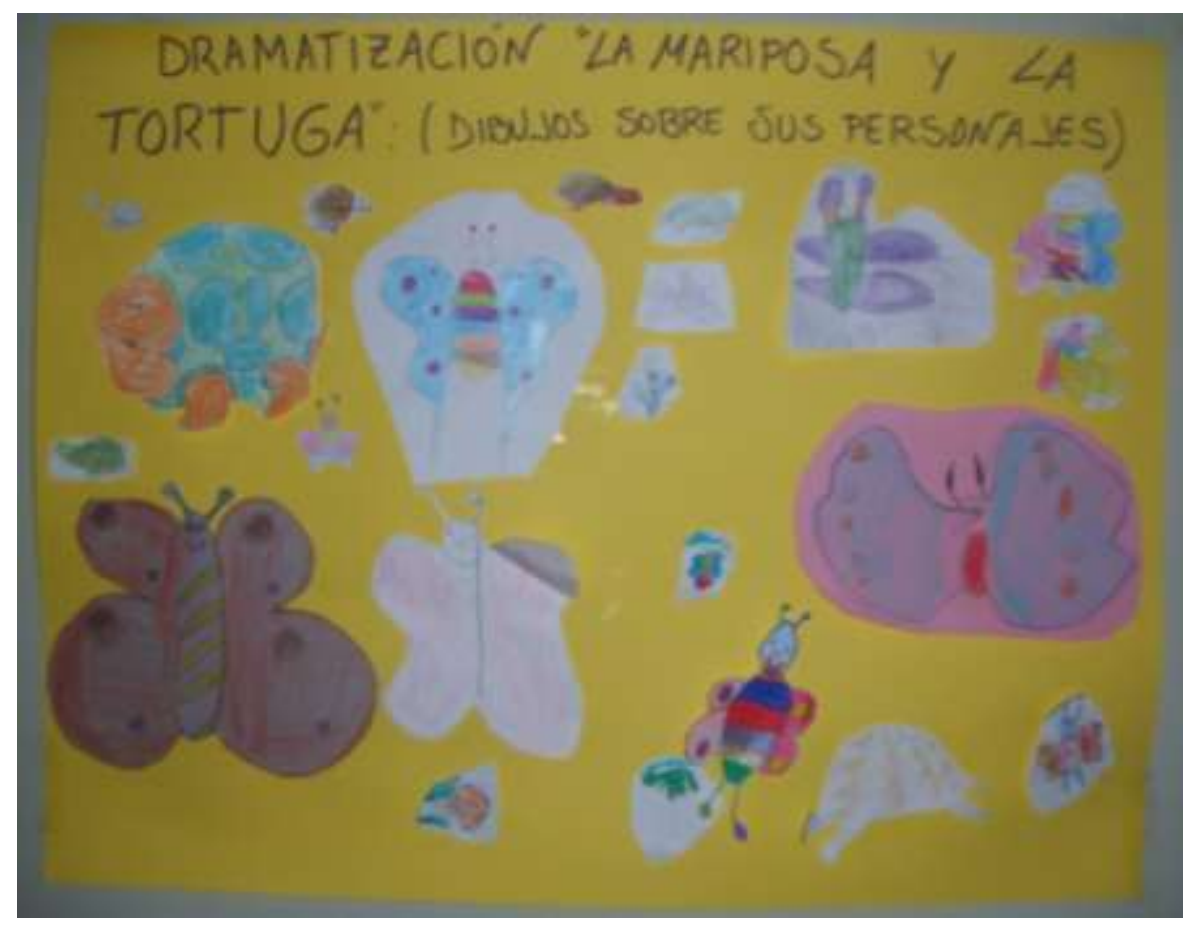




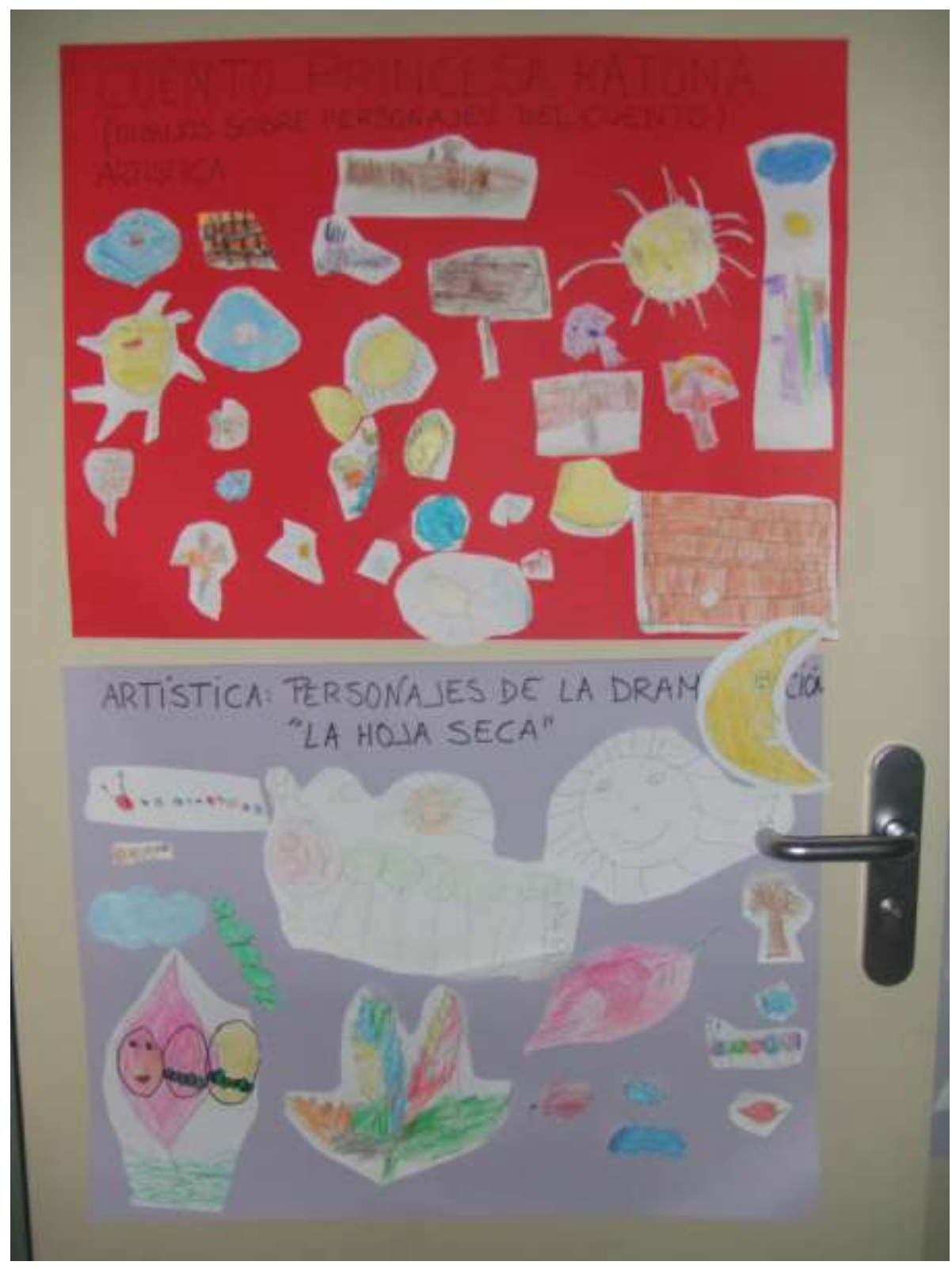

Como se puede observar, mediante estos murales el niño se siente como verdadero protagonista de su aprendizaje y disfruta con su creación y, lo más importante, con las de los demás. Después de la elaboración conjunta del "periódico-mural", los niños contaron la experiencia que habían recreado en sus dibujos con mayor facilidad.

Es una práctica muy recomendable tanto en la escuela infantil como en la primaria, además de por lo anteriormente dicho, porque desarrolla la creatividad, ya que en sus dibujos representan toda su imaginación sobre, por ejemplo, el cuento que ha escuchado, es decir, es este caso el alumno convierte las palabras que llegan a sus oídos en dibujos hechos por sus manos y procesados por su mente teniendo en cuenta siempre su personalidad.

\section{A MODO DE CONCLUSIONES}

Resulta obvio que la sociedad actual demanda que se trabaje de manera cooperativa para conseguir las metas propuestas. En los centros educativos el trabajo cooperativo no sólo de los alumnos sino también de los padres, familiares y profesores es muy 
enriquecedor y gratificante. En este sentido, resulta imprescindible la colaboración de los padres y familiares de los alumnos en la escuela.

Como hemos podido ver en este trabajo, son numerosas las acciones que pueden llevarse a cabo para conseguir que el individualismo quede en un segundo plano dando paso al trabajo colaborativo y en equipo para el fomento de los logros en cooperación con los demás. El sentido de comunidad que aflora al trabajar cooperativamente ofrece una riqueza especial al proceso de enseñanza-aprendizaje. De este modo se evitarán las incongruencias de la educación ya que el alumno obtendrá respuestas educativas semejantes tanto en casa como en el centro escolar.

Por otro lado, recordar que es muy importante que los futuros ciudadanos aprendan a trabajar en equipo desde la edad escolar para que de esta manera puedan aplicar las habilidades que se desarrollan a su vida en la sociedad. Por este motivo la relación cooperativa entre profesorado y familias resulta imprescindible ya que los alumnos la tomarán como referente y modelo a seguir por ellos mismos.

Para finalizar, animar a que este clima cooperativo tome especial relevancia en el ámbito escolar y que el papel de ponerse en el lugar del otro o empatía sea una cualidad a trabajar en las aulas y en los diferentes hogares de los alumnos. Trabajando de manera conjunta se facilitará el camino al éxito educativo de todos los implicados.

\section{BIBLIOGRAFÍA}

BRUNO CIARI (1981). Nuevas técnicas didácticas. Barcelona: reforma de la escuela.

COMELLAS, M. J. (2009). Educar en la comunidad y en la familia. Acompañando a las familias en el día a día. Valencia: Nau Libres.

COMELLAS, M. J. (2009). Familia y escuela: compartir la educación. Barcelona: Graó.

CAMPIGLIO, A. y RIZZI, R. (Dir.) (1997). Cooperar en clase. Ideas e instrumentos para trabajar en el aula. Sevilla: Publicaciones MCEP.

GARCÍA CARMONA, M. y SOLA MARTÍNEZ, T. (2010). En MANZANARES MOYA, A. (2010) (Ed.). Organizar y dirigir en la complejidad. Instituciones educativas en evolución. Madrid: Wolters Kluwer Educación.

LÓPEZ HERNÁNDEZ, A. (2007). 14 Ideas Clave. El trabajo en equipo del profesorado. Barcelona: Graó.

RUÉ, J. (1989). El trabajo cooperativo por grupos. En Cuadernos de Pedagogía, 170, mayo, pp. 18-21.

RUÉ, J. (1998). El aula: un espacio para la cooperación. En MIR, C. (Coord.); CASTELEIRO, J. M., CASTELLÓ, T., CIRERA, I., GARCÍA, M. T., JORBA, A., LECIÑENA, M., MOLINA, L., PARDO, A. M., RUÉ, J. TORREDEMER, M. y VILA, I. (1998). Cooperar en la escuela. La responsabilidad de educar en la democracia. Barcelona: Graó. 